\title{
XII. On the emission of polarized light by fluorescent gases
}

\section{R.W. Wood}

To cite this article: R.W. Wood (1908) XII. On the emission of polarized light by fluorescent gases, Philosophical Magazine Series 6, 16:91, 184-189, DOI: $10.1080 / 14786440708636498$

To link to this article: http://dx.doi.org/10.1080/14786440708636498

曲 Published online: 21 Apr 2009.

Submit your article to this journal $\llbracket$

Џ Article views: 5

Q View related articles $\sqsubset$

Citing articles: 5 View citing articles 5 


\section{$\left[\begin{array}{ll}184 & ]\end{array}\right.$}

XII. On the Emission of Polarized Light by Fluorescent Gases. By R. W. Woon, Professor of Experimental Physics, Johns Hopkins University*.

DREVIOUS attempts to detect polarized light in the 1 emission of fluorescent vapours have yielded negative results. Schmidt's observations were made with a nicol only, and he failed to detect any difference in the brilliancy of the fluorescence as the prism was rotated.

I have always felt that the apparent absence of polarization was difficult to reconcile with the theory that the fluorescence is merely a re-emission by electrons vibrating synchronously with the waves of the exciting light. Quite recently I have attacked the problem anew, and by carefully studying the conditions have succeeded in getting as much as 30 per cent. of polarized light in the resonance radiation of sodium and potassium vapour. The polarization of light produced in this way appears to be a wholly new effect, and its study throws considerable additional light upon the mechanism of radiation.

The phenomenon was first detected in the case of potassium vapour by means of a Savart plate which is capable of showing two per cent. of polarization. It was immediately picked up in the fluorescence of sodium and iodine, and it is probable that other vapours will be found to show it as well.

The apparatus used in the work consisted of a steel tube with a lateral branch brazed to its centre for the observation of the fluorescent light at an angle of $90^{\circ}$ with the exciting beam. This tube was used in the earlier work upon fluorescence, before the expedient of "end-on" examination bad been adopted. The metal was contained in a small retort also brazed to the large tube, immediately below the lateral observation-tube (fig. 1). The light of the arc was focussed

Fig. 1.

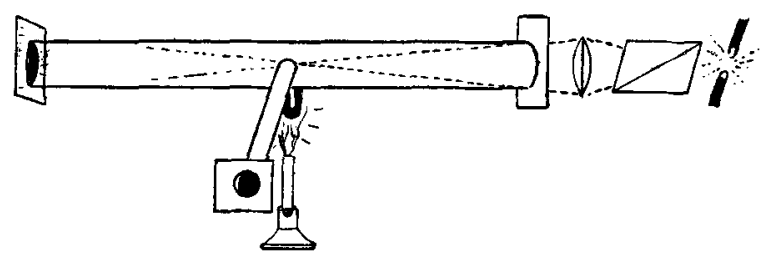

by a large lens at the centre of the large tube immediately in front of the lateral branch. Owing to the sensitiveness of the Savart plate it was necessary to eliminate all other possible

* Communicated by the Author. 
sources of polarization, such as fog or mist in the tube. Exhaustion with a Gaede mercurial pump while the tube was heated was sufficient to completely banish all trace of fog.

The complete absence of $f_{0} g$ can be told by illuminating the vapour with an intense beam of light embracing the spectrum region comprised between wave-lengths 5400 and 5700 , obtained by prismatic dispersion. Such a beam is incapable of exciting any fluorescence, and if fog is absent the cone of light is absolutely invisible when viewed through the lateral tube. The Savart fringes were very distinct, and the percentage of polarization was determined by compensating it with a pair of glass plates which could be turned about on a vertical axis furnished with a graduated circle. This method gives by far the most accurate results when we are dealing with small percentages of polarization. Cornu's method, which depends upon the unequal brilliancy of two images seen through a Rochon or other double-image prism, cannot be used when there is less than twenty-five or thirty per cent. of polarized light present, for with less than this amount it is difficult or impossible to detect any difference in the brilliancy of the two images. This is true at all events for the green fluoreseent light of sodium vapour: The first measurements were made with the exciting light polarized (electric vector) vertically.

It was found that the polarization was strongest when the temperature of the tube was comparatively low, i.e. when the fluorescence first appeared. At a higher temperature, with very bright fluorescence, compensation was secured with a single plate at an angle of $59^{\circ}$, or with two plates at an angle of $47^{\circ}$. Taking the refractive index of the glass as 1.52, and making use of the formula which expresses the ratio of the amplitudes of the components of the vibration in and perpendicular to the plane of incidence

$$
\frac{\mathrm{D}_{k}}{\mathrm{D}_{p}}=\frac{\mathrm{E}_{s}}{\overline{\mathrm{E}_{p}}} \cos ^{2}(\phi-\chi)^{*}
$$

we find the percentage of polarization to be 20 .

At the lowest temperature consistent with a fairly bright fluorescence compensation was secured with two plates at in angle of $52^{\circ}$. This gives us 30 per cent. of polarized light. The change in the intensity of the fluorescent spot when viewed through a slowly revolving nicol was easily observed in this case, and a separate determination was made by Cornu's method, which gave 28 per cent. of polarized light.

$$
\text { * 'Drude's Optics,' page 265, 1st edition. }
$$


If the exciting beam was polarized with its electric vector horizontal, no trace of the Savart fringes could be detected, which was to be expected as everything was then symmetrical about the line of sight.

If the exciting light was unpolarized to start with the fluorescent light was found to be polarized, but not to the same extent, the percentage in each case being one half of its former value, which was also to be expected. The question at once arose as to why we bave only a partial polarization of the fluorescent light when we start with plane-polarized light.

As I have already shown (Phil. Mag., May 1908) excitation of the vapour with monochromatic light causes it to emit this same wave-length and a large number of other wave-lengths, the spectrum exhibiting in the most typical case a number of very sharp lines spaced at nearly equal intervals along a normal spectrum. It at once occurred to me to see whether the polarization was confined to the line corresponding to the exciting line, for it seemed not impossible that the electron vibrating in synchronism with the exciting light might emit light which was completely plane-polarized, whereas the other disturbed electrons might emit unpolarized light. The vapour was accordingly excited with the light of the cadmium arc, and the spectrum examined with a small spectroscope furnished with a nicol prism. It was found, however, that the polarization was quite as strong in all parts of the spectrum as it was at the lines coinciding in position with the exciting lines. As a check on this observation the following experiment was then tried. The light from the arc was passed through a filter which removed everything above wave-length $5000, i . e$. which transmitted a deep blue light. In front of the Savart polariscope I placed a dense screen of aurantia which cut off everything below 5000. This removed all the wave-lengths from the fluorescent spectrum which were to be found in the exciting light, transmitting, however, the upper end of the fluorescent spectrum. This light was found to be strongly polarized, the fringes appearing quite as distinct as in the absence of the screens. It will be observed that this experiment completely eliminates all possible sources of error, such as polarization produced by fog or by reflexion from the back wall of the tube.

The D lines appear in the fluorescent spectrum stimulated by white light, and the spectroscope showed that they were polarized to about the same degree as the rest of the spectrum. As I have shown, the D lines can also be caused to appear by stimulation with blue light, but in this case they are too faint to make a study of their polarization possible. 
The foregoing experiments prove that we must seek elsewhere for the cause of the partial polarization. The decrease in the amount of polarization with increasing temperature made me think that the increased density of the vapour might have something to do with the phenomenon, in other words, that the depolarizing factor might be the molecular collisions.

To test this hypothesis a small amount of nitrogen was introduced into the tube, sufficient to raise the pressure to $12 \mathrm{mms}$. The number of collisions must now be vastly in excess of what obtains with sodium vapour in vacuo, for in this case the actual pressure of the metallic vapour is probably never in excess of a millimetre or two, if it is as great as that. In fact I now feel sure that the partial pressure of the sodium vapour can never be greater than the pressure of the residual gas in the tube. In my earlier work I overestimated the density of the vapour, and imagined that it must possess a viscosity, but I am now convinced that this is not so.

The polarization did not appear to be decreased by the presence of the nitrogen, which made it seem probable that molecular collision was not the depolarizing agency. Temperature appears to be the only remaining factor, and I am inclined to attribute the depolarization to the rotation of the molecule while it is traversing its mean free path. The speed of rotation will increase with the temperature, for the energy of a gas is partly energy of translation and partly energy of rotation, the ratio being constant. (Sodium is, however, regarded as monatomic, and the relation may not hold.)

That there is a depolarizing agent is probable from the most elementary theory. If we assume the electrons to be free to move in any direction, under the influence of the electric forces in the light-waves, it seems probable that with a polarized beam of exciting light, we should have planepolarized light emitted by the vapour in directions making an angle of $90^{\circ}$ with the exciting beam. If the stimulating light were plane-polarized to start with, there would be no fluorescent emission at $90^{\circ}$ in directions parallel to the electric vector in the exciting light. In other words, the vapour would behave in much the same way as a fog composed of very fine particles, though the physical processes involved would be quite different in the two cases.

Let us now assume that the electron is able to move along a straight line, the position of which is fixed within the molecule. While it is quite improbable that this condition actually holds, the conception of it will enable us to see how the introduction of constrained motion will decrease the amount of polarized light emitted by the vapour. We can 
imagine as a rough model of our molecule a hollow sphere with a wire passing through its centre, along which a bead can slide. Consider the polarized exciting waves as travelling along the $x$ axis towards the origin (fig. 2), with their

Fig. 2.

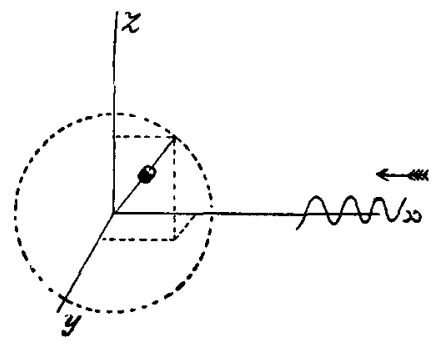

direction of vibration parallel to the $z$ axis. It is clear that the bead will vibrate with the greatest amplitude when the direction of the wire is also parallel to the $z$ axis. If the wire lies in the $x y$ plane no vibration will occur. In other positions there will be vibrations of greater or less amplitude, according to the component of the force in the light which is directed along the wire. We may represent the fluorescent gas by an enormous number of these vibrators with their wires pointing in all possible directions. The vibrations of the beads along the wires can each be resolved into two components, one parallel to the $x$ axis, the other parallel to the $z$ axis, and the integrated effects of these components, or rather of their squares, will give us the measure of the intensity of the emitted radiation vibrating parallel to the $x$ and $z$ axes. These integrals stand in the ratio of 6 to 2 , which means, since the total intensity is 8 , and their difference is 4 , that we are to expect a polarization of 50 per cent. with a vibration direction parallel to the $z$ axis. By experiment we find only 30 per cent.

As the percentage of polarization appears to decrease as the temperature of the tube is raised, it is possible that at lower temperatures than any which can be employed satisfactorily, the percentage may be bigher. As to possible depolarizing agencies, rotation of the molecule would doubtless act in this way if the electrons continued to emit light after the cessation of the incident light, or-expressing it in terms of our imaginary model-if the bead on the wire continued to vibrate after the wire turned into the $z y$ plane. It must be remembered, however, that sodium is regarded as monatomic, and the question arises as to whether we can ascribe 
much of the energy of the gas to rotation of the molecule. Another possible depolarizing factor is what may be termed secondary fluorescence, or flinorescence excited, not by the primary ray but by the resonance radiation of the gas. Lord Rayleigh has considered the possibility of an analogous action in his treatment of the theory of the colour of the sky and its polarization. The fluorescent light is of course rather feeble in comparison with the exciting light, but it must be remembered that its frequencies are just right, and the radiation from one molecule would without doubt be able to excite vibrations in a neighbouring one.

The discovery of the polarization of light by fluorescing gases will perhaps give us a satisfactory theory of the solar corona.

The radial polarization of the corona amounts to about 11 per cent., its spectrum is continuous, though some observers have found faint indications of the Fraunhofer lines, and it appears to be cold, that is no deflexions are obtained with the bolometer. Polarization is usually ascribed to scattering by small particles, the continuous spectrum to an emission by larger particles (white hot), while the apparent absence of radiant heat cannot very well be reconciled with the presence of white-hot particles. If we regard the corona as a cloud of mixed metallic vapours fluorescing under the terrific radiation from the sun, there seem to be fewer contradictions. 'l'he polarization is just about what we should expect, a continuous spectrum, at least with all resolving powers which can be brought to bear, would be almost certain, and there is no inherent difficulty in explaining the absence of radiant heat. The presence of faint traces of the Fraunhofer lines could be ascribed to light from the sky, which is largely due to light from the exposed edge of the sun. The so-called coronium line, and other bright lines peculiar to the corona, may be merely fluorescent lines of well-known elements. As I have shown already, the fluorescent lines of sodium bear no relation to any of the lines excited by other means. It appears to me to be even possible that the Fraunhofer lines are produced by the absorption of this outlying cloud of vapour rather than by a comparatively shallow layer as is usually supposed. These are of course questions that can be more intelligently discussed by those engaged in work upon solar physics, and I am merely drawing attention to a new factor that may possibly be introdnced to advautage. 